\title{
MusicApp: um aplicativo para apoio às crianças autistas nas aulas de musicalização
}

\author{
David W. F. Lima ${ }^{1}$, Rodrigo C. Pereira ${ }^{1}$, Marcos P. S. Farias ${ }^{1}$, Thais H. C. Castro ${ }^{1}$, \\ Lury J. Souza ${ }^{2}$, Jhessyka de A. Freitas ${ }^{3}$ \\ ${ }^{1}$ Instituto de Informática - Universidade Federal do Amazonas (UFAM) \\ Av. General Rodrigo Octávio, 6200, Coroado, 69077-000 - Manaus - AM - Brasil \\ ${ }^{2}$ Faculdade DeVry Martha Falcão \\ Manaus, AM - Brasil \\ ${ }^{3}$ Fundação Centro de Análise, Pesquisa e Inovação Tecnológica (FUCAPI) \\ Manaus, AM - Brasil \\ \{david.lima,rcp,mpsf, thais\} @icomp.ufam.edu.br, \{luuryjs, \\ jhessyka\} doutlook.com
}

\begin{abstract}
This work aims at presenting a prototype for a multimodal app for children with autism who are enrolled in a music class, as a way of broadening their learning opportunities for the music keys and tempo identification. We present the concepts supporting the MusicApp development, showing screenshots for three different modals and emphasizing the Android prototype, which is ready for further tests.
\end{abstract}

Resumo. Este trabalho tem como objetivo apresentar um protótipo de aplicativo multimodal para crianças autistas frequentando aulas de musicalização, como forma de ampliar a aprendizagem de identificação de notas musicais e tempo. Apresentamos os conceitos que suportam o desenvolvimento do MusicApp, mostrando telas para três modos diferentes de interface e enfatizando o protótipo para Android, o qual está pronto para testes mais completos.

\section{Cenário de uso}

Com a popularização dos computadores, smartphones, tablets e mesas multimídia, considerando o uso desses artefatos cada vez mais frequente em atividades cotidianas, pesquisadores de diversas áreas como, Computação, Música, Medicina e Psicologia, começaram a desenvolver artefatos computacionais para auxiliar pessoas com algum tipo de limitação, seja física ou cognitiva, nas atividades diárias [Lima e Castro, 2012].

Pesquisas relatam artefatos desenvolvidos para pessoas com Transtorno do Espectro do Autismo (TEA), que faz parte dos Transtornos Globais do Desenvolvimento (TGD) que é um grupo de síndromes que apresentam dificuldades na interação social, comunicação e imaginação [Lima e Castro, 2012], [Ribeiro et al. 2013], [Silva et al. 2013], [Sitdhisanguan et al. 2012].

Nesse contexto, algumas aplicações foram desenvolvidas para auxiliar nas aulas de musicalização e musicoterapia. Uma das propostas de utilização da musicalização é 
V Congresso Brasileiro de Informática na Educação (CBIE 2016)

Anais dos Workshops do V Congresso Brasileiro de Informática na Educação (CBIE 2016)

encontrada no trabalho de Pietrowicz et al. (2009) que demonstra a estrutura aberta para a criação, uso distribuído e colaborativo da prática de violino.

Para Gattino (2012), musicalização para crianças autistas pode auxiliar no desenvolvimento de habilidades sociais, emocionais, cognitivas e comunicacionais. Lima (2013) afirma que a musicoterapia pode possibilitar mudanças significativas no ambiente educacional da criança autista.

Baseado nessa perspectiva e em uma experiência com projeto de musicalização de crianças autistas através da prática de violino, no contexto do protótipo aqui apresentado, foram desenvolvidas sessões de prática de violino exclusivas para crianças autistas, com o auxílio de recursos computacionais como aplicativos (apps) para tablet. Dos apps pesquisados para apoiar a musicalização, os mais utilizados durante as sessões foram o "Pequeno Mozart" [Barbosa, 2012] e o "Juno's Musical ABCs" [Burton e Pearsall, 2016], respectivamente. Foram pesquisados e testados diversos outros apps, mas nenhum correspondeu às necessidades das crianças atendidas, principalmente pela complexidade de imagens na tela. A Tabela 1 identifica esses apps.

Tabela 1. Apps pesquisados e testados

\begin{tabular}{|c|c|c|}
\hline Nome do App & Website & Plataforma \\
\hline Rhythm Trainer & http://www.therhythmtrainer.com/ & Flash \\
\hline Clapping Music & http://clappingmusicapp.com/ & iPad \\
\hline $\begin{array}{l}\text { Rhythm Cat - Learn } \\
\text { To Read Music }\end{array}$ & $\begin{array}{l}\text { https://play.google.com/store/apps/details?id=net.gamy } \\
\text { a.rhythm }\end{array}$ & Android \\
\hline My Rhythm & http://www.myrhythm-app.com/ & iPad \\
\hline $\begin{array}{l}\text { Rhythm Training } \\
\text { (Sight Reading) }\end{array}$ & $\begin{array}{l}\text { https://itunes.apple.com/us/app/rhythm-training- } \\
\text { sightreading/id517988587?mt=8 }\end{array}$ & iPad \\
\hline Music Pal App & http://www.musicpalapp.com/ & iPad \\
\hline $\begin{array}{l}\text { Morton Subotnick's } \\
\text { Pitch Painter }\end{array}$ & $\begin{array}{l}\text { https://itunes.apple.com/us/app/morton-subotnicks- } \\
\text { pitch-painter/id519738403?ls=1\&mt=8 }\end{array}$ & iPad \\
\hline $\begin{array}{l}\text { DoReMi 1-2-3: Music } \\
\text { for Kids-A Fingerprint } \\
\text { Network App }\end{array}$ & $\begin{array}{l}\text { https://www.commonsensemedia.org/app- } \\
\text { reviews/doremi-1-2-3-music-for-kidsa-fingerprint- } \\
\text { network-app }\end{array}$ & iPad/Android \\
\hline $\begin{array}{l}\text { MusicTutor } \\
\text { LeiaMúsica }\end{array}$ & $\begin{array}{l}\text { https://play.google.com/store/apps/details?id=es.virtual } \\
\text { code.musictutor.sightread }\end{array}$ & iPad \\
\hline Juno's Musical ABCs & $\begin{array}{l}\text { https://www.commonsensemedia.org/app- } \\
\text { reviews/junos-musical-abcs }\end{array}$ & iPad \\
\hline $\begin{array}{l}\text { Five Little Musical } \\
\text { Monkeys }\end{array}$ & http://appcrawlr.com/ios/five-musical-monkeys-hd & iPad \\
\hline $\begin{array}{l}\text { Little Musician - } \\
\text { Rhythm }\end{array}$ & $\begin{array}{l}\text { https://itunes.apple.com/us/app/little-musician- } \\
\text { rhythm/id819185458?mt=8 }\end{array}$ & iPad \\
\hline $\begin{array}{l}\text { Rhythm Sheep - learn } \\
\text { music }\end{array}$ & $\begin{array}{l}\text { https://play.google.com/store/apps/details?id=turskaga } \\
\text { mes.rhythmsheep.free }\end{array}$ & Android \\
\hline
\end{tabular}

Após um ano de sessões de musicalização foi identificada a necessidade de se ampliar as noções de tempo e identificação de notas musicais, pois as crianças atendidas apresentaram muitas dificuldades relacionadas a essas noções, dificultando seu avanço 
V Congresso Brasileiro de Informática na Educação (CBIE 2016)

Anais dos Workshops do V Congresso Brasileiro de Informática na Educação (CBIE 2016)

na prática de violino. Portanto, foi desenvolvido o protótipo "MusicApp" para apoiar atividades presenciais de prática de ritmos e identificação de notas musicais.

\section{Desenvolvimento}

O Grupo de Sistemas Inteligentes (GSI) do Instituto de Computação da Universidade Federal do Amazonas desenvolve projetos que envolvem desenvolvimento e avaliação de ferramentas e ambientes que usam o conhecimento em situações reais, especialmente em interfaces adaptativas, tecnologia assistiva e no apoio e mediação do processo ensino-aprendizagem. Os recursos presentes no laboratório possibilitaram a prototipação do aplicativo MusicApp.

O processo de desenvolvimento utilizado foi no contexto de uma pesquisa participativa, onde os potenciais usuários (crianças autistas e professores de musicalização) e seus representantes (pais e terapeutas) testaram diversos apps, definindo assim as necessidades específicas para esse contexto. Isso foi realizado como parte das atividades de um projeto de pesquisa, em que as crianças participavam de sessões semanais de musicalização e violino.

O protótipo MusicApp foi desenvolvido como aplicativo para smartphones baseados na plataforma Android (a partir da versão 5.0). $O$ ambiente de desenvolvimento utilizado foi o Android Studio versão 2.1.2 utilizando as linguagens de programação Java e C. Para validação do protótipo, o mesmo foi testado inicialmente no simulador Android Virtual Device (AVD), onde foi possível simular o uso em diversos dispositivos (smartphones e tablets) e configurações. Além da simulação, três diferentes smartphones das marcas Samsung e Sony foram utilizados com o objetivo de validar e identificar possíveis erros.

\section{Apresentação do Software}

Conforme mencionado anteriormente, o MusicApp é um protótipo desenvolvido para crianças autistas que fazem aula de musicalização. O protótipo possui três níveis: o primeiro nível trabalha com o conceito de percepção melódica; o segundo trabalha com tempo e compasso; e o terceiro nível trabalha, simultaneamente, com os conceitos vistos nos níveis anteriores.

Ao iniciar o protótipo, a criança deverá jogar o primeiro nível. A primeira tela que aparece é a "Ouça a nota musical" (Figura 1a), onde a criança deverá clicar na opção "Ouvir". Após clicar, o som da nota musical será tocado e a opção correspondente ao som deverá ser escolhido (Figura 1b). O protótipo não possui feedback negativo. Para o funcionamento correto do protótipo, o mesmo deverá ser utilizando somente na orientação horizontal do smartphone. 
V Congresso Brasileiro de Informática na Educação (CBIE 2016)

Anais dos Workshops do V Congresso Brasileiro de Informática na Educação (CBIE 2016)
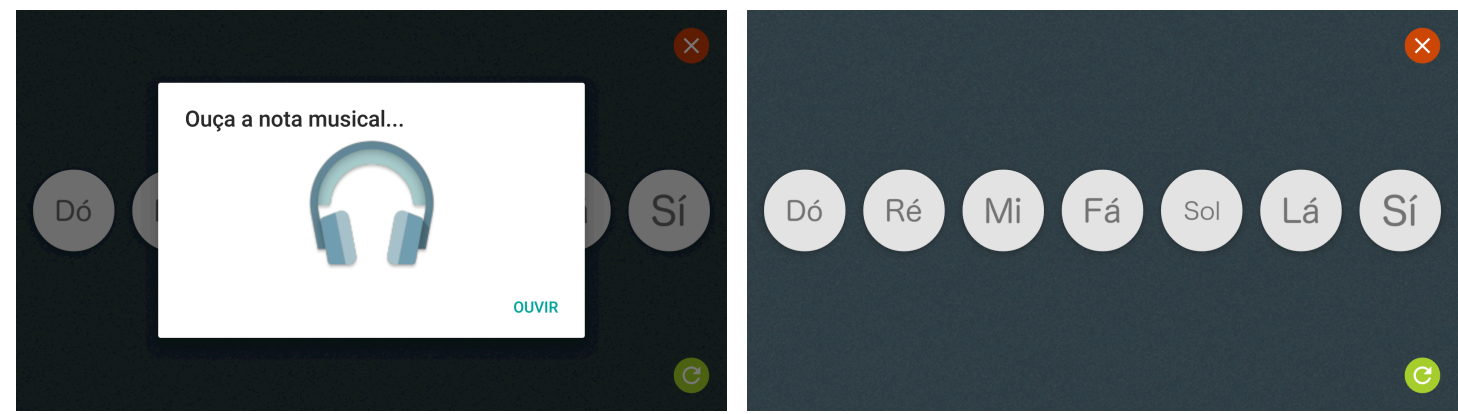

Figura 1. a) tela para ouvir a nota musical; b) tela para escolher a nota musical.

Ao acertar cinco vezes a nota musical, a criança irá para o segundo nível que consiste em acertar o tempo musical. O fluxo das atividades é similar ao primeiro nível. A Figura 2a mostra a tela "Ouça o tempo musical" e a Figura $2 b$ mostra os botões com os ícones que representam os tempos musicais. Para avançar para o próximo nível, a criança deverá acertar também cinco vezes.
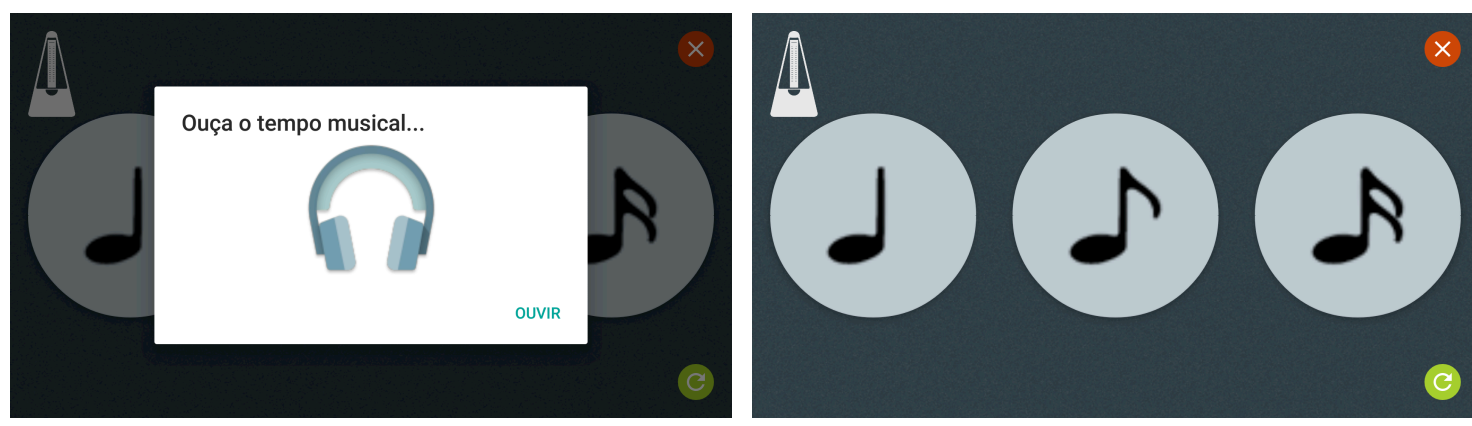

Figura 2. a) tela para ouvir o tempo musical; b) tela para escolher o tempo musical.

O último nível aborda a percepção musical, onde são trabalhados conceitos vistos nos níveis anteriores. A criança deverá ouvir a nota e o tempo musical (Figura 3a). Para acertar, a criança deverá escolher a nota e o tempo musical correspondente (Figura 3b). Quando a criança acerta o tempo musical, os botões referentes ao mesmo são ocultados (Figura 4a) e quando acertar a percepção melódica, os mesmos também são ocultados (Figura4b). A criança também deverá acertar cinco vezes.
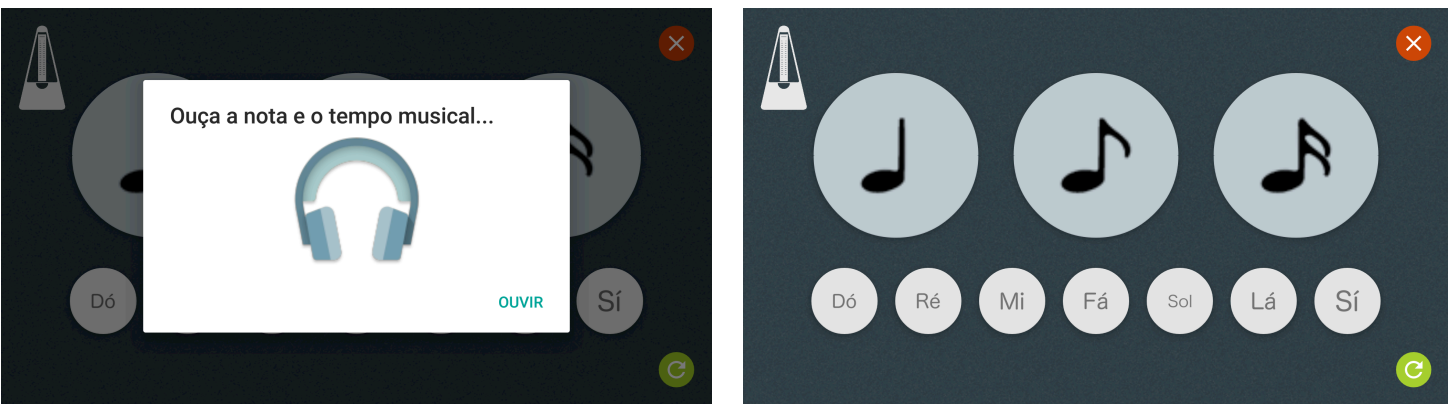

Figura 3. a) tela para ouvir a nota e o tempo musical; b) tela para escolher 0 tempo e a nota musical. 

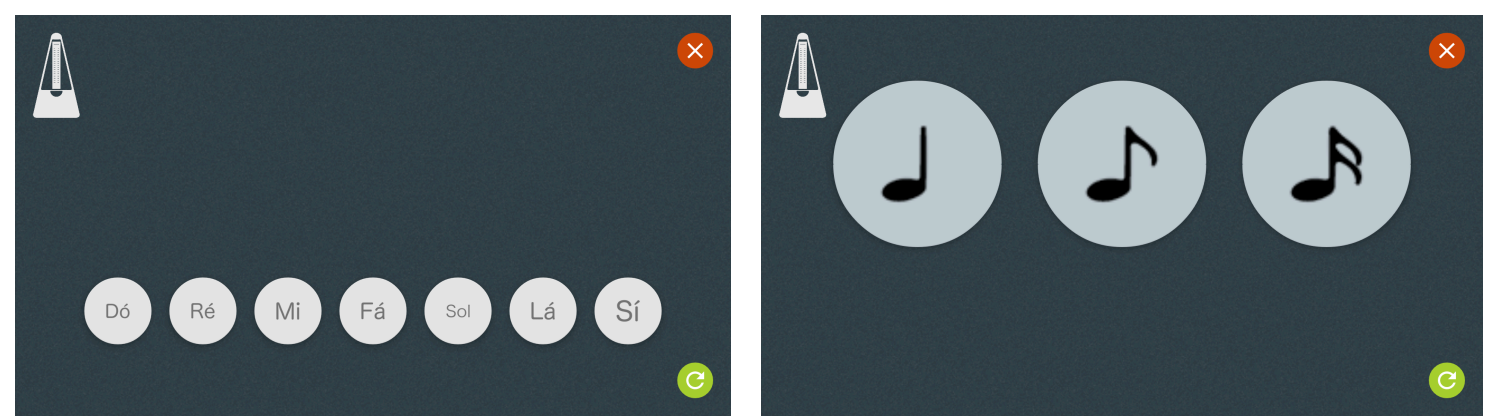

Figura 4. a) tela com os botões do tempo musical ocultos; b) tela com os botões das notas musicais ocultos.

Em todos os níveis a criança poderá escutar o som novamente através do clique no botão verde (botão a direita na parte inferior). Caso queira sair do MusicApp, deverá clicar no botão vermelho (botão a direita na parte superior).

O MusicApp também tem como objetivo ser multimodal. Para isso, uma versão do app está sendo implementada em iOS e como um protótipo tangível e a função "Professor" está sendo implementada na versão em Android. Tal função tem como objetivo auxiliar o professor de musicalização nas aulas e integrar as versões implementadas em Android, iOS e tangível. A Figura 5 mostra uma tela presente na versão em iOS. A referida imagem corresponde a mesma descrição e funcionalidade presente na Figura $3 b$.

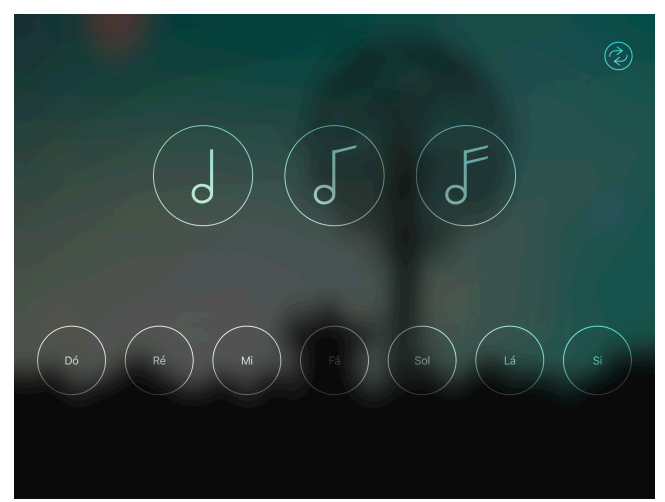

Figura 5. Versão inicial do MusicApp para iOS.

Conforme mencionado anteriormente, o MusicApp também está sendo implementado na forma tangível com as mesmas funcionalidades das versões em Android e iOS. A Figura 6 mostra o protótipo do MusicApp tangível.

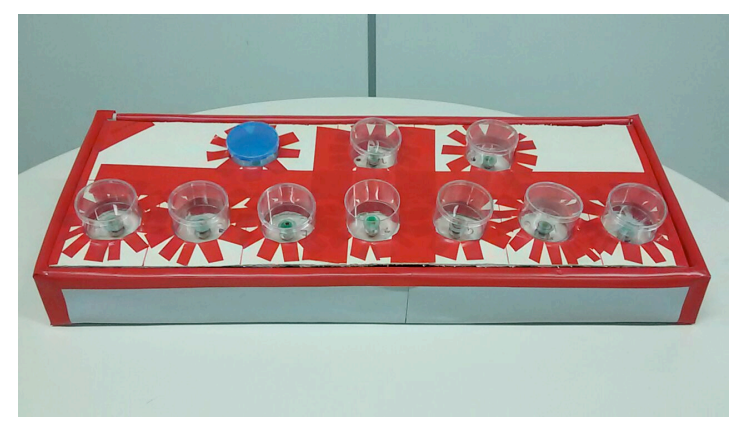

Figura 6. Versão inicial do MusicApp tangível. 
V Congresso Brasileiro de Informática na Educação (CBIE 2016)

Anais dos Workshops do V Congresso Brasileiro de Informática na Educação (CBIE 2016)

O projeto de interface do MusicApp está sendo elaborado por estudantes de design com o apoio de especialistas considerando recomendações de interface para crianças com deficiência intelectual. A Figura 7 mostra uma visão geral sobre esse projeto de interface.

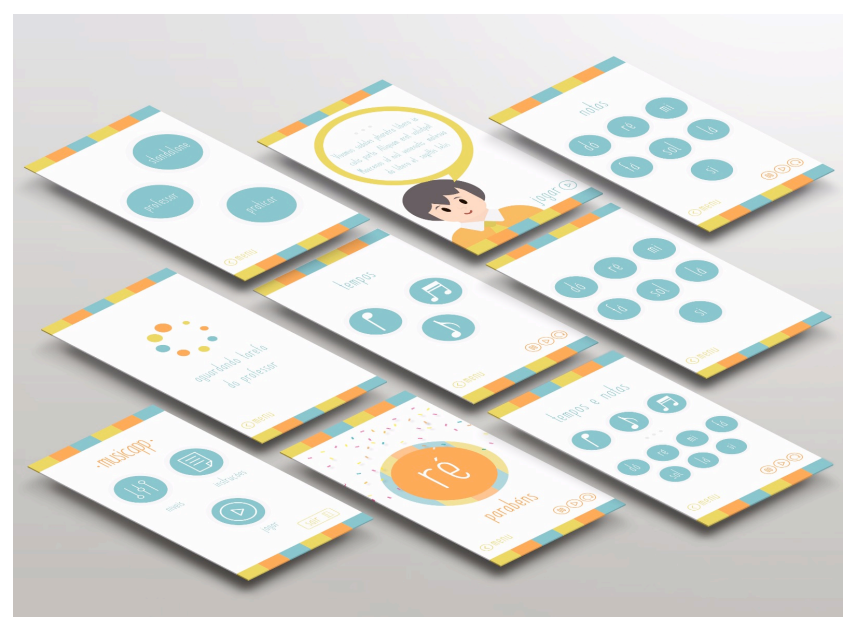

Figura 7. Visão geral sobre o projeto de interface do MusicApp.

O protótipo na plataforma Android pode ser obtido através do seguinte endereço http://migre.me/ufn37. Um vídeo explicativo sobre o protótipo pode ser visualizado no endereço https://youtu.be/UiRfgE-CEF0.

\section{Considerações finais}

Atualmente o protótipo está sendo reavaliado por especialistas com o objetivo de se utilizar em aulas presenciais de musicalização. Algumas funcionalidades também estão sendo implementadas, como o modo Professor, onde o mesmo poderá definir previamente a atividade que a criança deverá desenvolver. Outro ponto que está sendo trabalhado é a melhoria da interface com a implementação de feedback positivo e a implementação do protótipo na plataforma iOS e tangível.

Como resultados da validação, os especialistas (professores de musicalização) constataram que o protótipo tem potencial para ser utilizado em aulas de musicalização inclusivas ou para crianças autistas. A próxima etapa é a realização de testes experimentais com crianças autistas que frequentam cursos de musicalização em instituições locais. Outro objetivo é avaliar possíveis benefícios pedagógicos que o uso das versões do MusicApp pode trazer para o processo de ensino-aprendizagem das crianças autistas.

\section{Referências}

Barbosa, S. M. T. (2012). "Software pequeno Mozart: uma porta para a música: um estudo de caso na paralisia cerebral". Trabalho de Conclusão de Curso. Escola Superior de Educação de Paula Franssinetti, Porto.

Burton, S. L., and Pearsall, A. (2016). "Music-based iPad app preferences of young children. Research Studies in Music Education", 38(1), 75-91.

Gattino, G. S. (2012). "Musicoterapia aplicada à avaliação da comunicação não verbal de crianças com transtornos do espectro autista: revisão sistemática e estudo de 
V Congresso Brasileiro de Informática na Educação (CBIE 2016)

Anais dos Workshops do V Congresso Brasileiro de Informática na Educação (CBIE 2016)

validação". Tese de Doutorado em Saúde da Criança e Adolescente, Universidade Federal do Rio Grande do Sul.

Lima, D.; Castro, T. (2012). "Music Spectrum: A Music Immersion Virtual Environment for Children with Autism". In Proceedings of the 4th International Conference on Software Development for Enhancing Accessibility and Fighting Info-exclusion (DSAI 2012). Procedia Computer Science, Elsevier, vol.14, 111-118.

Lima, D. (2013). "Music Spectrum: imersão musical para crianças com autismo". $88 \mathrm{f}$. Dissertação (Mestrado em Informática) - Universidade Federal do Amazonas, Manaus.

Ribeiro, J. C.; Falcão, T. (2009) "Mundos virtuais e identidade social: processos de formação e mediação através da lógica do jogo". Tecnologias de Comunicação e Subjetividade, 16 (1), 84-96.

Silva, G. F. M.; Salgado, L. C. C.; Raposo, A. B. (2013). "Metáforas de Perspectivas Culturais na (re) definição de padrões de colaboração de um jogo de multi-toque para usuários com autismo". In Proceedings of the 12th Brazilian Symposium on Human Factors in Computing Systems (IHC '13), 112-121.

Sitdhisanguan, K.; Chotikakamthorn, N.; Dechaboon, A.; Out, P. (2012). "Using tangible user interfaces in computer-based training systems for low-functioning autistic children”. Personal Ubiquitous Comput. 16, 2: 143-155.

Pietrowicz, M.; Mcgrath, R.E.; Smith, B.; Garnett, G. (2009). "Transforming Human Interaction with Virtual Worlds". In Workshop on Computational Creativity Support at CHI 2009. Boston. 